\title{
Advantages of a new modulation scheme in an optical self-mixing frequency-modulated continuous-wave system
}

\author{
Daniel Nordin \\ Kalevi Hyyppä \\ Lulea University of Technology \\ Division of Industrial Electronics \\ S-971 87 Lulea, Sweden \\ E-mail: danieln@sm.luth.se
}

\begin{abstract}
A new frequency-modulated continuous wave modulation scheme, which gives correct results even when the Doppler shift is larger than the frequency difference associated with the range, is presented and tested with a tunable laser diode and fiber-based system. By inserting a constant frequency region in the modulation scheme, both the magnitude and the sign of all beat frequencies can be determined. When they are known, the correct frequency difference as a result of the range can be calculated. This new scheme gives more freedom when choosing the modulation parameters of the laser, because increasing the modulation frequency, and/or the frequency sweep, to avoid ambiguities resulting from a large Doppler shift no longer becomes necessary. This is especially useful when using a somewhat cheaper laser diode source, since the maximum obtainable modulation frequency and frequency sweep can be somewhat limited. The suggested modulation scheme makes it possible to use some laser diodes in an application they otherwise would not be suited for. (C) 2002 Society of Photo-Optical Instrumentation Engineers. [DOI: 10.1117/1.1467063]
\end{abstract}

Subject terms: lidar; frequency-modulated continuous wave; frequency modulation; Doppler effect.

Paper 010077 received Mar. 2, 2001; revised manuscript received Aug. 23, 2001; accepted for publication Nov. 29, 2001.

\section{Introduction}

Frequency-modulated continuous wave (FMCW) is a range and radial velocity measuring technique well known from the field of radar applications. The reflected part of an outgoing frequency modulated wave $(w 1)$ is mixed with a local oscillator wave $(w 2)$, and as a result the frequency difference between the two signals can be obtained. If the frequency modulation is chosen as a triangular wave, both radial velocity and range of the target can be determined from the frequency difference, usually referred to as beat frequency. By using a tunable laser as the source, with the light frequency as the carrier frequency and a photodiode as detector, the beat frequency can be extracted directly from the photodiode current. This phenomena, usually referred to as the "self-mixing effect," is due to the fact that the photodiode delivers a current proportional to the squared sum of the two electrical fields $w 1$ and $w 2$. The most common type of lasers used are diode lasers of DFB or DBR type, $\mathrm{CO}_{2}$, or Nd:YAG. ${ }^{1-9}$ Systems can be built either as classical open Michelson interferometers or with optical fibers. Our intended application is indoor range measurements of distances up to $10 \mathrm{~m}$ against a diffuse reflecting surface.

\section{General FMCW Theory}

\subsection{Self-Mixing Effect}

If the received electrical field is denoted as $E_{r} \cos \left(\omega_{r} t\right.$ $+\theta_{r}$ ), and the local oscillator electrical field is denoted
$E_{l o} \cos \left(\omega_{l o} t+\theta_{l o}\right)$, the optical power received by the photodiode, ignoring reflections at the photodiode, will equal

$\Phi(t)=\frac{A}{Z}\left[E_{r} \cos \left(\omega_{r} t+\theta_{r}\right)+E_{l o} \cos \left(\omega_{l o} t+\theta_{l o}\right)\right]^{2}$,

where $A$ is the active area and $Z$ is the characteristic impedance of the medium in front of the detector. By expanding Eq. (1) and removing terms with frequencies too high for the photodiode to respond to, the current from the diode can be expressed as

$$
\begin{aligned}
i(t) & =\mathfrak{R} \Phi(t)+i_{b} \\
& =\frac{\mathfrak{R} A}{Z}\left[\frac{E_{l o}^{2}}{2}+\frac{E_{r}^{2}}{2}+E_{l o} E_{r} \cos \left(\omega_{b} t+\theta_{r}-\theta_{l o}\right)\right]+i_{b},
\end{aligned}
$$

where $f=2 \pi \omega_{b}$ is the beat frequency, $\mathfrak{R}$ is the responsivity of the detector, and $i_{b}$ is the direct current in the detector, which can include both dark current and current generated by background light. It should also be pointed out that Eq. (2) is only valid when the two waves have the exact same polarization states, and that polarization mismatch degrades the performance.

\subsection{Modulation}

The simplest modulation scheme consists of a sawtooth wave that only allows range measurements to a stationary target. The more sophisticated FMCW scheme used today 
is displayed in Fig. 1. It has the advantage that both range $R$ and radial velocity can be calculated from two measured beat frequencies $f_{1}$ and $f_{2}$. The time $T_{e}=2 R / c$ in Fig. 1 represents an error interval, corresponding to the flight time to target and back, where no useful measurements can be made. If we assume that the range and velocity of the target are constant during one modulation period, $f_{1}$ and $f_{2}$ can be expressed as

$f_{1}=\left|f_{R}-f_{D}\right|$

$f_{2}=\left|-f_{R}-f_{D}\right|=\left|f_{R}+f_{D}\right|$,

where $f_{R}$ is the magnitude of the frequency difference due to the range and $f_{D}$ is the Doppler shift. If $\left|f_{D}\right|<f_{R}$, the frequency $f_{R}$ can be expressed as

$f_{R}=\frac{f_{1}+f_{2}}{2}$,

and $f_{D}$ as

$f_{D}=\frac{f_{2}-f_{1}}{2}$.

If $\left|f_{D}\right|>f_{R}$, the frequencies would instead have to be calculated from

$f_{R}=\left|\frac{f_{2}-f_{1}}{2}\right|$,

and
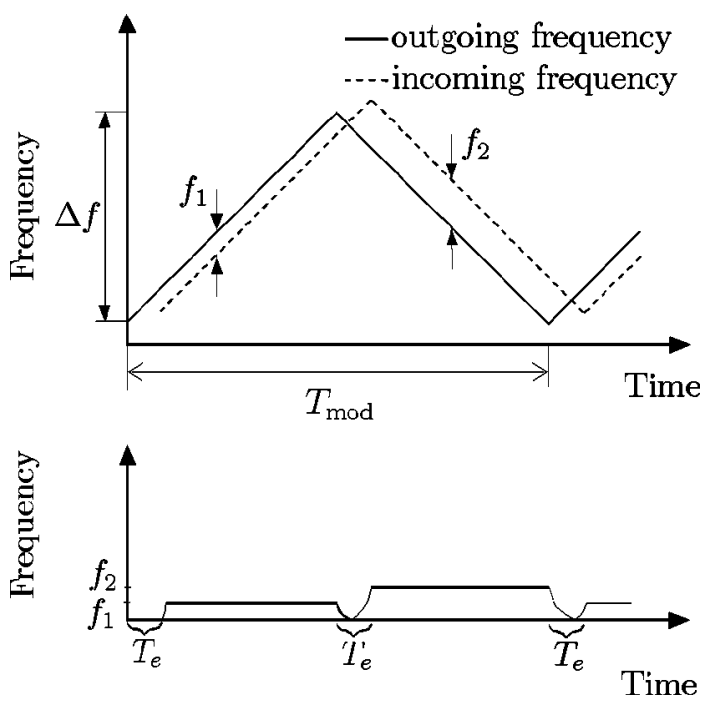

Fig. 1 The upper part shows the frequencies of the outgoing and incoming waves. The lower part shows the corresponding beat frequencies.
$f_{D}= \begin{cases}\frac{f_{2}+f_{1}}{2}, & f_{2}>f_{1} \\ -\left(\frac{f_{2}+f_{1}}{2}\right), & f_{2}<f_{1} .\end{cases}$

If we have determined the correct values of $f_{R}$ and $f_{D}$, the range and radial velocity can be calculated. Since the modulation parameters $T_{\text {mod }}$ and $\Delta f$ are known, $f_{R}$ will depend on the range according to

$f_{R}=\frac{4 R \Delta f}{c T_{\bmod }}$.

The range $R$ can then be calculated from

$R=\frac{c f_{R} T_{\mathrm{mod}}}{4 \Delta f}$

and the radial velocity $v_{t}$ of the target is given by ${ }^{10}$

$v_{t}=\frac{f_{D} \lambda}{2}$

where $\lambda$ is the optical wavelength. If the distance between source and target decreases, the sign of $f_{D}$, and hence $v_{t}$, will be positive.

\section{Problem Description and Background}

When using triangular modulation, it is commonly assumed that $\left|f_{D}\right|<f_{R}$ and the maximum allowed Doppler shift is hence upper bonded by $f_{R}$, since Eqs. (5) and (6) give incorrect results if $\left|f_{D}\right|>f_{R}$. To deal with larger Doppler shifts, it hence becomes necessary to increase $\Delta f$ and/or $f_{\text {mod }}=1 / T_{\text {mod }}$, thereby increasing the value of $f_{R}$ for a given range. However, depending on the laser source used, this may not be a practical solution. Both parameters are commonly upper bounded, and the performance will suffer if they are increased too much. One example of this is the one section DFB laser used in the experiments described in Sec. 6. In a one-section laser diode, the current modulation must be added to the bias current. If the laser is to operate in single mode, the current may not drop below a certain value, and increasing the current modulation too much also increases the nonlinearity of the optical sweep. If the optical frequency modulation is mostly due to thermal effects, as appears to be the case with our laser, increasing the modulation frequency $f_{\text {mod }}$ too much also decreases $\Delta f$. Others have also reported this effect, as well as the problem caused by larger Doppler shifts. ${ }^{11}$ Some laser diodes also have a built in low-pass filter to protect the diode from spikes in the drive current. This, of course, also limits $f_{\text {mod }}$.

\section{New Modulation Scheme}

To overcome the explained problem, we have to know if $\left|f_{D}\right|>f_{R}$. Our suggested solution is to include a constant frequency region in the modulation scheme, as shown in Fig. 2. It would also be possible to insert the constant region at the peek of the modulation triangle. By inserting this region, $\left|f_{D}\right|$ can be measured separately. If $f_{2}>f_{1}$, the 

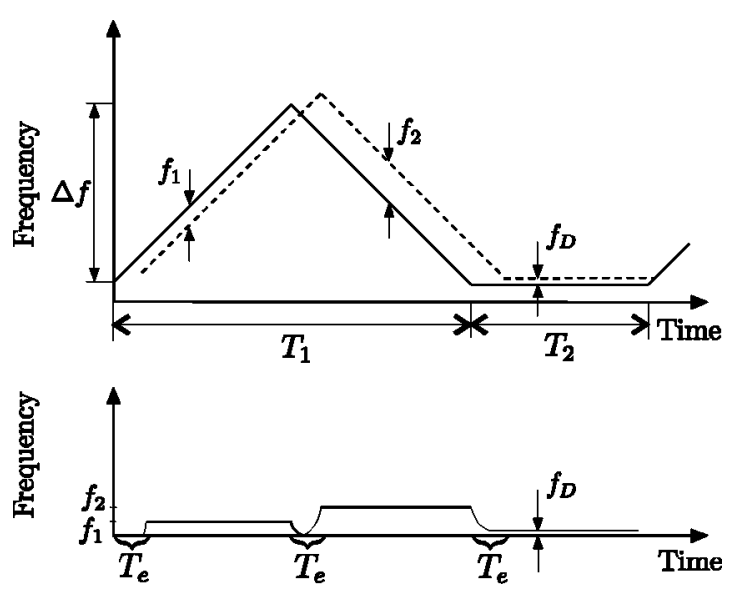

Fig. 2 The new modulation scheme with a constant frequency region.

sign of $f_{D}$ must be positive, and negative if $f_{2}<f_{1}$. The measured $f_{D}$ can be compared to the calculated one by inserting the measured values of $f_{1}$ and $f_{2}$ in Eq. (6). If the difference between the measured and calculated $f_{D}$ is large, we can assume that $\left|f_{D}\right|>f_{R}$, and $f_{R}$ can then be calculated from Eq. (7).

\section{Choice of Parameters}

If the suggested modulation scheme is used, the maximum allowed $f_{D}$ is no longer upper bounded by $f_{R}$, and as a result we have more freedom when choosing the $\Delta f$ and $T_{1}$ parameters. Note that $T_{1}$ in Fig. 2 corresponds to $T_{\text {mod }}$ in Fig. 1. Reducing $\Delta f$ and/or increasing $T_{1}$ decreases the frequency $f_{R}$ for a given range $R$, as seen in Eq. (9). Decreasing $f_{R}$ decreases the larger one of the two measured frequencies $f_{1}$ and $f_{2}$, and the required bandwidth of the detector can be reduced. By reducing $\Delta f$, it is easier to achieve linear modulation and avoid mode hopping by using the laser where the relation between current and frequency is as linear as possible. However, there is one major drawback associated with lowering $\Delta f$, since the theoretical spatial resolution $\Delta R$ is given by ${ }^{12}$

$\Delta R=\frac{c}{2 \Delta f}$

i.e., decreasing $\Delta f$ increases $\Delta R$.

The maximum achievable range will depend on $T_{1}$. If $T_{2} \geqslant T_{1} / 2$,

$R_{\max }<\frac{c T_{1}}{4}$

This indicates that if the maximum possible range for a selected value of $T_{1}$ is desired, measurements can only be taken near the turning points of the frequency modulation waveform, as shown in Fig. 3. However, commonly the largest realistic flight time is much smaller than the time required to establish linear modulation when the modulation changes from a negative to a positive derivative, or

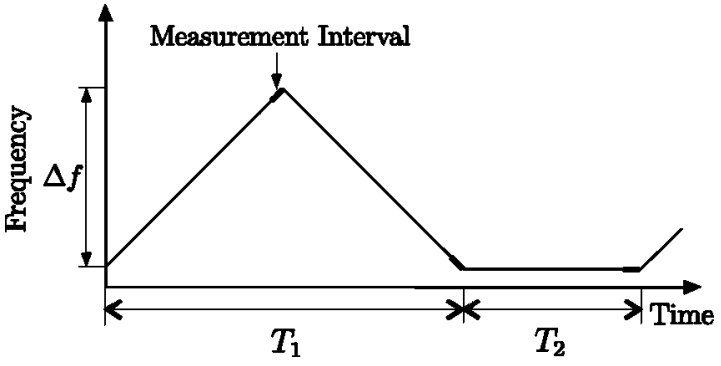

Fig. 3 Measurement intervals at maximum possible range.

vice versa. Hence that time, rather than the flight time, determines the start of the measurement interval. When the frequency difference between $f_{D}$ and $f_{R}$ is small, and either $f_{1}$ or $f_{2}$ approaches zero, the measurement interval also determines the lowest measurable beat frequency, since at least one period of the frequency must fit under the measurement interval. The time $T_{2}$ must be set large enough for the output laser frequency to stabilize and for a sufficient amount of periods of the Doppler shift to fit under the remaining time. Detecting smaller Doppler shifts requires a longer $T_{2}$. Depending on application and modulation parameters, it can also be advantageous not to include $T_{2}$ in each period. In a rapidly scanning system, the introduction of $T_{2}$ can lead to less accurate distance measurements, for instance to static walls inside a room.

\section{Measurement Setup}

Fiber-based systems are attractive because of their simplicity. Several other authors, for instance in Ref. 11, have built and reported on systems using a fiber optical coupler in a similar way, as we have done in our experiments. A second attractive alternative is to use a fiber optical circulator instead of a coupler. ${ }^{13}$ By using a circulator, the loss of optical power associated with the coupler, as well as other problems described later, can be avoided.

The test system we used is displayed in Fig. 4. One advantage with this type of system is that a minimum of alignment is required. A local oscillator can be achieved either by a separate reflector at the free fiber end or by utilizing the refractive index difference between fiber core and air at one of the two fiber ends. If we allow two reflections, one at each fiber end, to build up the local oscillator, the interference between the two beams can cause the local oscillator to vary in intensity. This kind of interferometer is very sensitive and responds to small changes in the fiber lengths, caused by, for instance, temperature and pressure changes. In this application, this effect is a disturbance. Including an isolator in the free fiber end would reduce it. Due to the coupler, we also lose a part of both the outgoing and reflected light. The isolator in front of the laser is required to keep reflected light from disturbing the laser.

Our system is built around a $10-\mathrm{mW}, 1.51-\mu \mathrm{m}$ onesection DFB laser, where $10 \mathrm{~mW}$ is the output power of the laser cavity, which leaves around $1 \mathrm{~mW}$ that propagates out of the collimated end in our system. We used nonpolarization maintaining single mode fibers and a 50/50 coupler. By modulating the drive current to the laser, variations in the current influence the cavity optical length, which results in a modulation of the optical output frequency. The cavity 


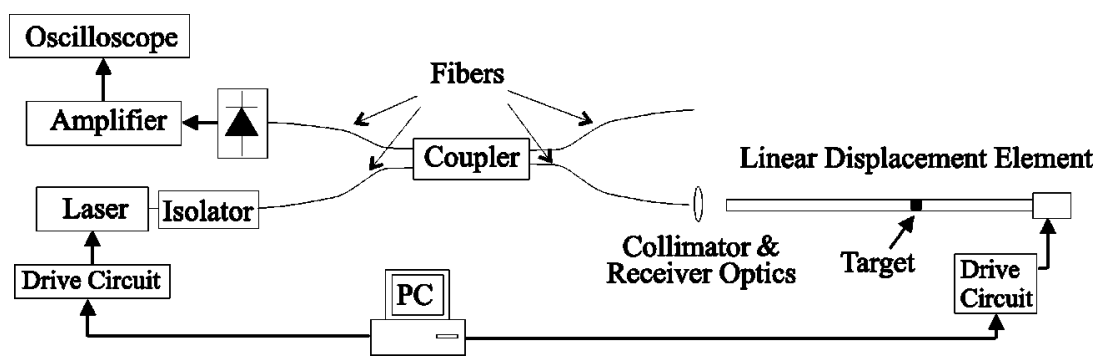

Fig. 4 Our measurement setup using a simple fiber-based system and tunable laser diode.

optical length is effected by temperature changes and changes in refractive index, both caused by the varying current. In our experiments, the current was swept using our suggested modulation scheme. As no special measures are taken to insure that the resulting optical modulation is linear, this will result in a slightly nonlinear modulation of the optical frequency. At our measuring distance of 0.5 to $1.0 \mathrm{~m}$, and with our choice of modulation parameters, the nonlinear optical sweep will cause the beat frequency to increase slightly during the modulation ramp. If the modulation amplitude is increased, the nonlinearity increases, as expected. Our system also lacks any form of temperature regulation.

The target was coated with retroreflective tape and moved back and forth between 576 to 976-mm distance measured to the fiber end. The movement was controlled using a linear displacement unit, and the laser was driven using voltage waveforms generated in a PC. The voltage was converted to current by our laser drive and protection circuit. The current was ramped from 91.54 to $97.51 \mathrm{~mA}$, and we picked $T_{1}=8.5 \mathrm{~ms}$ and $T_{2}=1.5 \mathrm{~ms}$. This current modulation was chosen as it gave a fairly linear ramping of the optical output frequency. The diode laser used in our setup has a built-in low-pass filter to protect the diode against spikes in the drive current. This low-pass filter and the fact that $T_{1}$ also influences $\Delta f$, which is common for our type of laser diodes since the optical frequency modulation is mainly caused by temperature effects, lead to our selected value of $T_{1}$. A decrease in $T_{1}$ otherwise resulted in a decrease of $\Delta f$. In our case, $T_{2}$ was chosen from the required time for the laser frequency to stabilize, and the Doppler shift was measured at the end of $T_{2}$. With a target speed of $0.05 \mathrm{~m} / \mathrm{s}$, the target will move approximately 0.5 mm during one measurement period $T_{1}+T_{2}$.

\subsection{Measurement Results}

Measurements were performed to verify the suggested modulation waveform and to investigate how the constant frequency region would effect the modulation. Our system is under development and the presented data should not be used to critically evaluate range and velocity measurement accuracy. The measurements also serve the purpose of illustrating how a Doppler shift would cause problems, even at relatively low velocities, if regular triangular modulation is used.

As we have a relatively low value of $\Delta f$, and a long $T_{1}$, the measured beat frequencies to a stationary target, $f_{1}$ and $f_{2}$ listed in Table 1 , will be fairly low. The $f_{1}, f_{2}$, and $f_{D}$ values in Table 1 were obtained by measuring the mean value of the last ten periods for each modulation ramp $N$ number of times. The clear difference between $f_{1}$ and $f_{2}$ appears to be caused by the insertion of the $T_{2}$ region, since the laser cavity temperature decreases during $T_{2}$. A slight difference between $f_{1}$ and $f_{2}$ is always present due to the triangular modulation form. This difference also increases with the amplitude of the current modulation. But with our modulation parameters and with $T_{2}=0$, the difference between $f_{1}$ and $f_{2}$ was negligible. From all ten listed values

Table 1 Measurement results from our laboratory system.

\begin{tabular}{|c|c|c|c|c|c|c|}
\hline Measurement & $\mathrm{N}$ & Mean $/ \mathrm{Hz}$ & Std.Dev./Hz & Std.Err./Hz & Mean $\Delta f / G H z$ & Calc. $\mathrm{R} / \mathrm{mm}$ \\
\hline$f_{1}$ at $576 \mathrm{~mm}$ & 10 & 4476 & 22.29 & 7.05 & 4.954 & 584.35 \\
\hline$f_{2}$ at $576 \mathrm{~mm}$ & 10 & 4394 & 22.95 & 7.258 & 4.863 & 573.55 \\
\hline$f_{1}$ at $676 \mathrm{~mm}$ & 10 & 5368 & 18.08 & 5.17 & 5.062 & 700.69 \\
\hline$f_{2}$ at $676 \mathrm{~mm}$ & 10 & 5120 & 22.4 & 7.095 & 4.828 & 668.32 \\
\hline$f_{1}$ at $776 \mathrm{~mm}$ & 10 & 6016 & 79.47 & 25.13 & 4.942 & 785.27 \\
\hline$f_{2}$ at $776 \mathrm{~mm}$ & 10 & 5793 & 63.22 & 19.99 & 4.759 & 756.16 \\
\hline$f_{1}$ at $876 \mathrm{~mm}$ & 10 & 6811 & 64.43 & 20.38 & 4.957 & 889.04 \\
\hline$f_{2}$ at $876 \mathrm{~mm}$ & 10 & 6558 & 45.28 & 14.32 & 4.773 & 856.02 \\
\hline$f_{1}$ at $976 \mathrm{~mm}$ & 10 & 7525 & 56.97 & 18.02 & 4.915 & 982.24 \\
\hline$f_{2}$ at $976 \mathrm{~mm}$ & 10 & 7327 & 89.07 & 28.17 & 4.786 & 956.40 \\
\hline$f_{D}$ at $0.05 \mathrm{~m} / \mathrm{s}$ & 20 & 66352 & 1081 & 241.8 & - & - \\
\hline
\end{tabular}


of $\Delta f$, we obtained an overall mean $\Delta f$ of $4.885 \mathrm{GHz}$. From this $\Delta f$ and the measured mean values of $f_{1}$ and $f_{2}$, we calculated the approximate distance using Eq. (10). In this case both the difference in $\Delta f$ on the up and down ramps and the nonlinear optical sweep are assumed to be the main contributors to the overall error. Normally one would assume a constant distance during one period and use both $f_{1}$ and $f_{2}$ to calculate the range, which brings the calculated values closer to the real values for our measurements.

In the experiments outlined before, we performed measurements to a fixed target at a known distance. This was done to calibrate the system and to obtain an approximate value of $\Delta f$. In the lab, $\Delta f$ appears to be pretty stable, since the temperature in the room is approximately constant. In our setup we also have a fixed beat frequency resulting from internal reflections in the system. Part of the local oscillator wave is reflected at the fiber-photodiode junction. This reflection will travel back through the coupler and a second reflection will occur at the fiber airjunction. When that reflection reaches the photodiode, it will be mixed with the local oscillator wave. The obtained beat frequency can then be used to calculate $\Delta f$, since the fiber length involved is known. By monitoring that beat frequency, it should be possible to continuously calibrate the system. In a system lacking this type of reflection, for instance a system built around a fiber optical circulator, a fixed reference beat frequency can be obtained by inserting a coupler before the circulator and have that signal travel down a reference path of known length. That signal can then be mixed with the local oscillator signal using a second coupler.

Some simultaneous measurements of $f_{1}, f_{2}$, and $f_{D}$ against a moving target were also performed. For the case in Fig. 5, we obtained $f_{1}=59.88 \mathrm{kHz}, f_{2}=73.26 \mathrm{kHz}$, and $f_{D}=65.79 \mathrm{kHz}$. Since $f_{2}>f_{1}$, the sign of $f_{D}$ must be positive. The edgy and fat appearance of channel 2 is a result of the oscilloscope display. Inserting the measured values of $f_{1}$ and $f_{2}$ in Eq. (6) gives $f_{D}=6.69 \mathrm{kHz}$. Since the measured $f_{D}$ is significantly different compared to the calculated one, we can assume that $\left|f_{D}\right|>f_{R}$ and Eq. (7) gives $f_{R}=6.69 \mathrm{kHz}$. From our calculated mean $\Delta f$, this would correspond to a distance of $873.32 \mathrm{~mm}$. In this measurement, the target was moved between 776 and $876 \mathrm{~mm}$. The measurements verify the modulation scheme and show how it can avoid errors that can be problematic at short distances even for relatively low velocities. If regular triangular modulation were used, a laser source similar to the one in our test system would not be suitable for many types of applications. For instance, if the intended application is robotic navigation or just plane range scanning in an environment containing moving objects, even a relatively low velocity can result in $\left|f_{D}\right|>f_{R}$.

\section{Conclusion}

We have presented a new FMCW modulation scheme that avoids errors resulting from Doppler shifts larger than the frequency associated with the range. The modulation scheme was tested and verified using a tunable laser diode

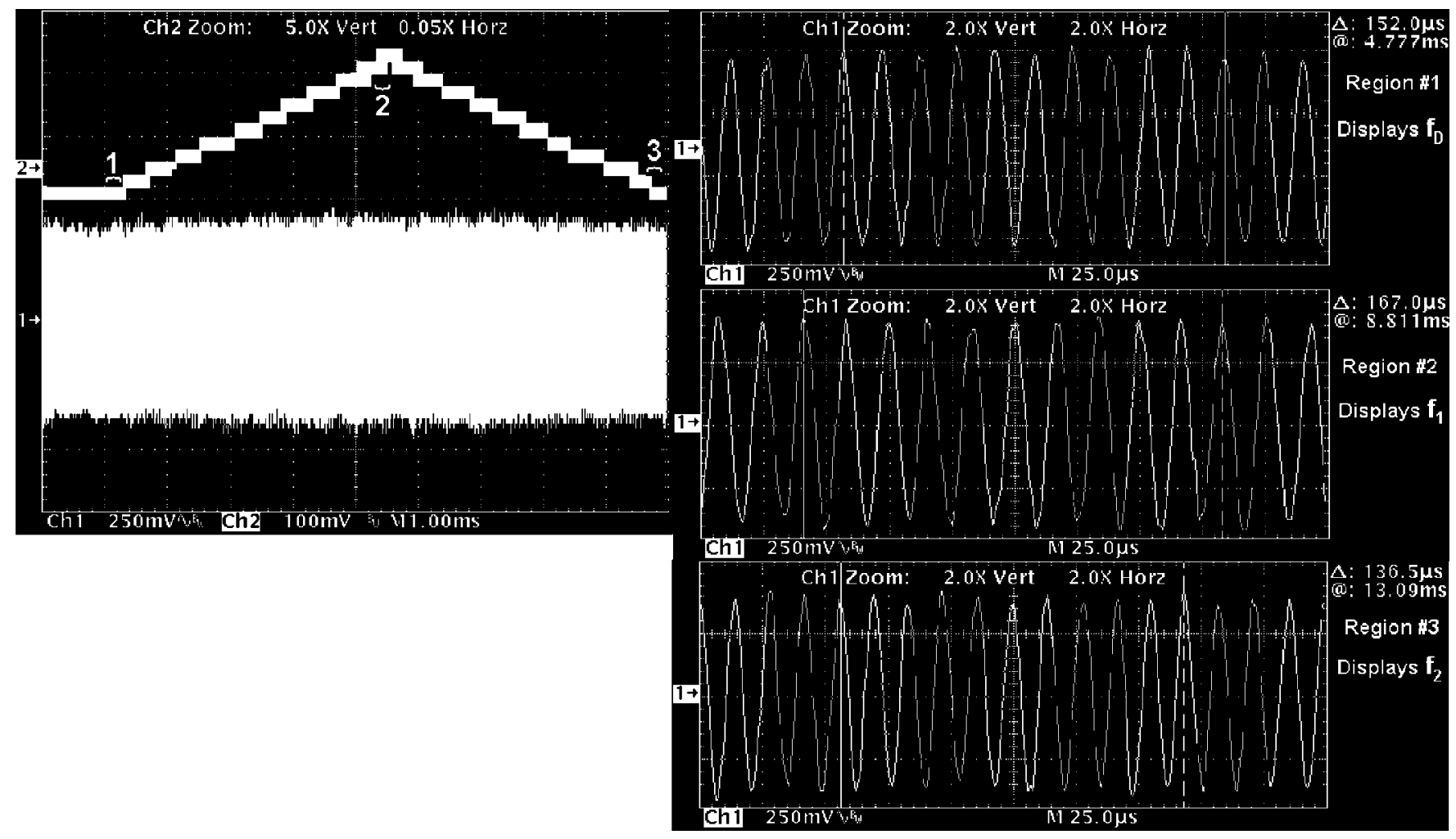

Fig. 5 Display of the simultaneous measurements of all three beat frequencies during one specific period. 
and a fiber-based system. One advantage to using this modulation scheme is that it no longer becomes necessary to increase $\Delta f$ and/or $f_{\text {mod }}$ to deal with larger Doppler shifts. This is especially useful when using a somewhat cheaper laser diode, since the maximum obtainable $\Delta f$ and $f_{\text {mod }}$ can be limited. The gained freedom when choosing the system parameters can also simplify both the detection task, by lowering the required bandwidth, and the task of achieving linear modulation by sacrificing spatial resolution. One disadvantage observed in our systems is an increased difference between the measured $f_{1}$ and $f_{2}$ as $T_{2}$ is inserted. In some systems, the drawback of this effect can be reduced by not including $T_{2}$ in each modulation period.

Our experimental system used to verify the modulation waveform is primitive and in an early stage of development. By replacing the coupler with a fiber optical circulator, improving the linearity of the optical sweep, and tuning both receiver optics and electronics, we aim to improve the performance of our system up to a point where measurements to diffuse targets at ranges up to $10 \mathrm{~m}$ can be performed. Continuous calibration can then be achieved by adding a reference path of known length to the fiber optical circulator-based system and mixing the reference signal with the local oscillator signal.

\section{Acknowledgments}

This research is supported by grant from Volvo Research Foundation, Volvo Educational Foundation, and Dr. Pehr G. Gyllenhammars Research Foundation.

\section{References}

1. T. Kubota, M. Nara, and T. Yoshino, "Interferometer for measuring displacement and distance," Opt. Lett. 12(5), 310-312 (1987).

2. A. R. Slotwinski, F. E. Goodwin, and D. L. Simonson, "Utilizing GaAlAs laser diodes as a source for frequency modulated continuous wave (FMCW) coherent laser radars," Proc. SPIE 1043, 245-251 (1989).

3. V. Shalini and S. V. Wayne, "Phase noise considerations in coherent optical FMCW reflectometry," J. Lightwave Technol. 11(10), 16941699 (1993).

4. R. Passy, N. Gisin, J. P von der Weid, and H. H. Gilgen, "Experimental and theoretical investigation of coherent OFDR with semiconductor laser source," J. Lightwave Technol. 12(9), 1622-1630 (1994).

5. A. Dieckmann and M.-C. Amann, "Frequency-modulated continuouswave (FMCW) lidar with tunable twin-guide laser diode," Proc. SPIE 2249, 22-30 (1994).
6. M. Millnert, C. Carlsson, D. Letalick, and I. Renhorn, "Robust coherent laser radar design and signal processing for vibrometry," Proc. SPIE 2748, 100-107 (1996).

7. U. Minoni, G. Scotti, and F. Docchio, "Wide range distance meter based on frequency modulation of an Nd:YAF laser," Opt. Eng. 35(7), 1949-1952 (1996).

8. C. Karlsson and D. Letalick, "Frequency modulation of a DFB laser diode for CLR applications," Proc. 9th Conf. on Coherent Laser Radar, pp. 156-159, Linköping (June 1997).

9. M. Nöding and M.-C. Amann, "Influence of nonlinear tuning characteristic of widely tunable laser diodes on FMCW-LIDAR performance," Proc. EOS 14th Topical Meeting on Optoelectronic Distancel Displacement Measurements and Applications, Nantes (July 1997).

10. K. J. Gåsvik, Optical Metrology, 2nd ed., pp. 43-44, John Wiley \& Sons, New York (1995).

11. C. J. Karlsson and F. A. Olsson, "Linearization of the frequency sweep of a frequency-modulated continuous-wave semiconductor laser radar and the resulting ranging performance," Appl. Opt. 38(15), 3376-3386 (1999)

12. A. J. Hymans and J. Lait, "Analysis of frequency modulated contiuous wave ranging system," Proc. IEEE 107B, 365-372 (1960).

13. R. L. Sebastian, R. B. Clark, D. L. Simonson, and A. R. Slotwinski, "Fiber optic coherent laser radar 3D vision system," Proc. SPIE 2348, 266-274 (1995)

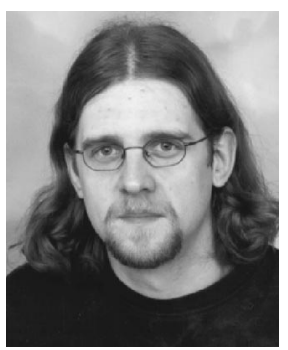

Daniel Nordin received his MS degree in electrical engineering from Luleå University of Technology in 1999. He is currently working towards the $\mathrm{PhD}$ degree at the EISLAB division at Luleå University of Technology. His main research interests include frequency modulation of diode lasers and optical ranging

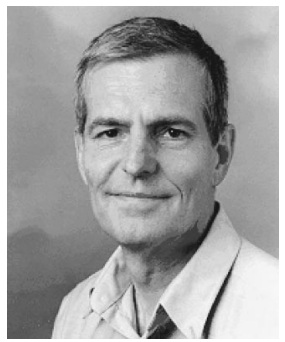

Kalevi Hyyppä received his MSEE from the Royal Institute of Technology in Stockholm, Sweden, in 1967. For several years he developed equipment for research on particle accelerators and rocket-borne instruments for plasma physics research in the ionosphere. After moving to Luleå University of Technology in 1975, he developed optoelectronic measurement equipment for industrial use. In 1991, he received the "Engineer of the Year" award in Sweden in recognition for successful development of an optoelectronic navigation system for mobile robots. He received his PhD from Luleå University of Technology in 1993. His current research interests are optoelectronics, step motors, and mobile robots. 